Research in Astron. Astrophys. 2012 Vol. X No. XX, 000-000

http://www.raa-journal.org http://www.iop.org/journals/raa

$\boldsymbol{R}$ esearch in

Astronomy and

Astrophysics

\title{
Measurements of Ca II Infrared Triplet Lines of Young Stellar Objects
}

\author{
Keiko Moto'oka ${ }^{1}$, Yoichi Itoh ${ }^{2}$ \\ 1 The Saga Prefecture Space \& Science Museum, 16351, Nagashima, Takeo, Takeo, Saga 843-0021, \\ Japan \\ 2 Nishi-Harima Astronomical Observatory, Center for Astronomy, University of Hyogo, 407-2, \\ Nishigaichi, Sayo, Hyogo 679-5313, Japan; yitoh@nhao.jp
}

Received 2012 June 12; accepted 2012 July 27

\begin{abstract}
Equivalent widths and line widths of Ca II infrared triplet emission lines were measured in high-resolution optical spectra of 39 young stellar objects. We found that the equivalent widths of the emission lines decrease with stellar evolution. It has been often claimed that strong chromospheric activity is generated by a dynamo process caused by fast rotation of the photosphere. However, we found no clear correlation between the strength of the Ca II lines and the stellar rotation velocity. Instead, we found that the objects with high mass accretion rates had stronger Ca II emission lines. This correlation supports the turbulent chromosphere model or the magnetic accretion theory for classical $\mathrm{T}$ Tauri stars. We also noticed that the equivalent widths of Ca II lines of transitional disk objects are one-tenth of those of classical $\mathrm{T}$ Tauri stars, even if the masses of the circumstellar disks are comparable.
\end{abstract}

Key words: stars: pre-main sequence — stars: emission lines

\section{INTRODUCTION}

The chromosphere is the region between the photosphere and the corona, through which energy is transferred via regular and irregular activities. The chromosphere and its associated events are well investigated for the nearest star, the Sun. The Hinode satellite obtained space- and time-resolved images of the solar chromosphere and revealed its energetic activity (e.g., Katsukawa et al. 2007).

For main-sequence stars, chromospheric activity is often discussed in relation to the stellar rotation. Noyes et al. (1984) found that objects with short rotation periods have strong Ca II H \& K emission lines, which are an indicator of chromospheric activity. They concluded that chromospheric activity is induced by the magnetic field generated by the dynamo process. The idea that chromospheres are magnetically driven is widely accepted for solar-mass main-sequence stars, and has also been applied to young stellar objects (YSOs). Neuhaeuser et al. (1995) found that weak-line T Tauri stars (WTTSs) have stronger X-ray emission than classical T Tauri stars (CTTSs). Because WTTSs have a faster mean rotational velocity than CTTSs, it is indicated that the strong dynamo process by fast rotation activates the coronal region of the WTTSs.

Another indication of activity of YSOs was reported by Hamann \& Persson (1992a), who carried out an optical spectroscopy of $53 \mathrm{~T}$ Tauri stars and $32 \mathrm{Herbig}$ Ae/Be stars. They detected narrow line emissions such as $\mathrm{Ca}$ II and $\mathrm{Mg}$ I. They interpreted these data to mean that the narrow lines are generated in stellar chromosphere. In addition to the narrow line component, Ca II infrared triplet (IRT) emission 
Table 1 Targets

\begin{tabular}{|c|c|c|c|c|}
\hline Object & Spectral Type $^{1}$ & Binary $^{2}$ & $R$ R-mag ${ }^{3}$ & Telescope \\
\hline \multicolumn{5}{|c|}{ Classical T Tauri stars } \\
\hline AA Tau & M0 & $\mathrm{S}$ & 11.80 & Keck/HIRES \\
\hline BP Tau & K7 & $\mathrm{S}$ & 13.38 & Keck/HIRES \\
\hline CW Tau & K5 & $\mathrm{S}$ & 11.75 & Keck/HIRES \\
\hline CY Tau & M2 & $\mathrm{S}$ & 12.50 & Keck/HIRES \\
\hline DF Tau & K5 & B & 13.34 & Keck/HIRES \\
\hline DG Tau & G & $\mathrm{S}$ & 11.40 & Keck/HIRES \\
\hline DK Tau & M0 & B & 11.08 & Keck/HIRES \\
\hline DL Tau & G & $\mathrm{S}$ & 11.85 & Keck/HIRES \\
\hline DO Tau & $\mathrm{G}$ & $\mathrm{S}$ & 12.30 & Keck/HIRES \\
\hline DR Tau & K4 & $\mathrm{S}$ & 10.68 & Keck/HIRES \\
\hline FN Tau & M5 & $\mathrm{S}$ & 13.48 & Keck/HIRES \\
\hline FP Tau & M2.5 & $\mathrm{S}$ & 12.13 & Keck/HIRES \\
\hline FX Tau & M4 & B & 12.40 & Keck/HIRES \\
\hline GI Tau & K5 & S & 12.15 & Keck/HIRES \\
\hline GK Tau & K7 & B & 11.58 & Keck/HIRES \\
\hline HL Tau & K9 & $\mathrm{S}$ & 10.63 & Hamann \& Persson \\
\hline HN Tau & K5 & B & 13.31 & Keck/HIRES \\
\hline LkCa 8 & M0 & $\mathrm{S}$ & 12.70 & Keck/HIRES \\
\hline RW Aur & G5 & B & 10.06 & Hamann \& Persson \\
\hline RY Tau & F8 & $\mathrm{S}$ & 10.20 & Hamann \& Persson \\
\hline T Tau & G5 & B & 9.19 & Hamann \& Persson \\
\hline XZ Tau & G & $\mathrm{B}$ & 13.56 & Hamann \& Persson \\
\hline
\end{tabular}

1: SIMBAD database

2: B: Binary, S: Single star. Leinert et al. (1993), Ghez et al. (1997), Kohler \& Leinert (1998), Sartoretti et al. (1998), Ireland \& Kraus (2008),

3: PPMXL catalog

lines often have a broad line component. This profile is well explained by the magnetospheric accretion model (e.g. Muzerolle et al. 1998).

We investigated the activity of YSOs by examining Ca II IRT emission. The Ca II IRT lines are one of the strongest emission lines in an optical spectrum of a low-mass YSO. They have only a small amount of interstellar extinction compared to $\mathrm{Ca}$ II $\mathrm{H} \& \mathrm{~K}$ lines in blue.

\section{OBSERVATIONS}

We carried out high-resolution optical spectroscopy of $12 \mathrm{~T}$ Tauri stars using the High Dispersion Spectrograph (HDS) mounted on the Subaru Telescope. The data were obtained on 2007 September 18 with the StdNIRb mode and the 0 "' 6 width slit. These instrument settings achieved a wavelength coverage of $6650-9360 \AA$ and a spectral resolution of $\sim 60,000$. The integration time for each object was between $600 \mathrm{~s}$ and $1500 \mathrm{~s}$.

We also used archived data of $27 \mathrm{~T}$ Tauri stars that were obtained with the High Resolution Echelle Spectrometer (HIRES) mounted on the Keck Telescope. The data were taken by S. E. Dahm on 2006 November 30, 2006 December 1, 2008 December 3, and 2008 December 4. The wavelength coverage was between $4800 \AA$ and $9380 \AA$ and the spectral resolution was $\sim 70,000$. The integration time for each object was between $300 \mathrm{~s}$ and $1200 \mathrm{~s}$. We also used the Ca II IRT measurements of 6 T Tauri stars reported in Hamann \& Persson (1992a). The objects we investigated are summarized in table 1.

We reduced the HDS data using the following standard steps: overscan subtraction, bias subtraction, flat fielding, removal of scattered light, extraction of a spectrum, wavelength calibration using a Th-Ar lamp, and continuum normalization. We used IRAF packages for all data-processing procedures. A detail description of the data-reduction method is presented in Takagi et al. (2011). The HIRES data were reduced with the Mauna Kea Echelle Extraction (MAKEE) package.

Emission lines of the Ca II IRT are superimposed on broad photospheric absorptions of Ca II. To construct spectra in which the equivalent widths of these emission lines are measured, we filled the 
Table 1 (continued)

\begin{tabular}{|c|c|c|c|c|}
\hline$\overline{\text { Object }}$ & Spectral Type $^{\perp}$ & Binary $^{2}$ & $\overline{R-\mathrm{mag}^{3}}$ & Telescope \\
\hline \multicolumn{5}{|c|}{ Transitional disk objects } \\
\hline CoKu Tau 4 & M1.5 & $\mathrm{B}$ & 12.10 & Keck/HIRES \\
\hline CX Tau & M1.5 & $\mathrm{S}$ & 12.63 & Keck/HIRES \\
\hline DM Tau & K5 & $\mathrm{S}$ & 13.61 & Keck/HIRES \\
\hline FO Tau & M2 & B & 14.00 & Keck/HIRES \\
\hline GM Aur & K5 & $\mathrm{S}$ & 11.02 & Keck/HIRES \\
\hline LkCa 15 & K5 & $\mathrm{S}$ & 11.43 & Keck/HIRES \\
\hline UX Tau & G5 & B & 10.30 & Keck/HIRES \\
\hline V773 Tau & $\mathrm{K} 2$ & B & 7.49 & Keck/HIRES \\
\hline V836 Tau & K7 & $\mathrm{S}$ & 12.70 & Keck/HIRES \\
\hline \multicolumn{5}{|c|}{ Weak-line T Tauri stars } \\
\hline HBC 374 & K7 & $S$ & 11.21 & Subaru/HDS \\
\hline HD 283716 & K0 & $\mathrm{S}$ & 9.79 & Subaru/HDS \\
\hline NTTS $032641+2420$ & K1 & $\mathrm{S}$ & 11.60 & Subaru/HDS \\
\hline NTTS 041559+1716 & K7 & $\mathrm{S}$ & 11.50 & Subaru/HDS \\
\hline NTTS 042417+1744 & K1 & $\mathrm{S}$ & 10.20 & Subaru/HDS \\
\hline RX J0405.3+2009 & K1 & $\mathrm{S}$ & 9.57 & Subaru/HDS \\
\hline RX J0409.2+1716 & M1 & $\mathrm{S}$ & 12.50 & Subaru/HDS \\
\hline RX J0438.6+1546 & $\mathrm{K} 2$ & S & 10.27 & Subaru/HDS \\
\hline RX J0452.5+1730 & K4 & $\mathrm{S}$ & 11.50 & Subaru/HDS \\
\hline RX J0459.7+1430 & K4 & $\mathrm{S}$ & 10.80 & Subaru/HDS \\
\hline V410 Tau & K4 & B & 8.45 & Hamann \& Persson \\
\hline V819 Tau & K7 & S & 12.24 & Keck/HIRES \\
\hline V827 Tau & K7 & $\mathrm{S}$ & 11.39 & Subaru/HDS \\
\hline V830 Tau & M0-1 & $\mathrm{S}$ & 11.30 & Subaru/HDS \\
\hline
\end{tabular}

absorption features using a spectrum of a dwarf with the same spectral type. We obtained the dwarf spectra from the HIRES data archive. Because the majority of YSOs are fast rotators, the dwarf spectra were convolved with a Gaussian profile so that the FWHMs of the photospheric absorption lines were comparable to those of the YSO spectra. Each convolved dwarf spectrum was subtracted from the corresponding YSO spectrum, then add unity. This procedure mostly removed the broad Ca II absorptions as well as the other photospheric absorption lines. Although it is possible that veiling effects caused by circumstellar materials could also alter the YSO emission spectra, we did not compensate for this because the veiling effect is insignificant in the $I$-band (Bertout et al. 1988).

We measured the equivalent widths, line widths, and radial velocities of Ca II IRT emission lines by fitting the line profiles with a Voigt function. Before subtracting the dwarf spectra, we obtained the rotational velocity $(v \sin i)$ of each YSO by measuring the FWHM of an unblended photospheric absorption line of Ti at $8683 \AA$ A. For objects whose values were taken from Hamann \& Persson (1992a), the radial velocities of the Ca II IRT emissions and the photospheric rotational velocity are unknown.

\section{RESULTS}

Figure 1 shows example spectra from a CTTS, a transitional disk object, and a WTTS. In these examples, photospheric absorption lines are not subtracted. The spectrum of the CTTS shows the strongest and broadest emission lines for the Ca II IRT, completely filling the absorption components. The transitional disk object has narrow emission lines, and the WTTS shows weak emission lines. Table 2 lists the equivalent widths, the FWHMs, and the radial velocities computed from the emission lines as well as the rotational velocity of the photosphere, the mass accretion rate, and the mass of the circumstellar disk.

\section{DISCUSSION}

Our results indicate that the Ca II IRT emission lines weaken with increasing stellar age. Figure 2 shows the equivalent widths of the Ca II IRT emission lines as a function of the evolutional sequence of the 
Table 2 Measurements of Ca II infrared triplet lines

\begin{tabular}{|c|c|c|c|c|c|c|c|c|c|c|c|c|}
\hline \multirow[t]{2}{*}{$\overline{\text { Object }}$} & \multicolumn{3}{|c|}{$\begin{array}{c}\text { Equivalent width } \\
[\AA]]\end{array}$} & \multicolumn{3}{|c|}{$\begin{array}{l}\text { Line width } \\
{\left[\mathrm{km} \mathrm{s}^{-1}\right]}\end{array}$} & \multicolumn{3}{|c|}{$\begin{array}{c}\text { Radial velocity } \\
{\left[\mathrm{km} \mathrm{s}^{-1}\right]}\end{array}$} & \multirow[t]{2}{*}{$\begin{array}{c}v_{\text {rot }} \\
{\left[\mathrm{km} \mathrm{s}^{-1}\right]}\end{array}$} & \multirow[t]{2}{*}{$\begin{array}{c}\log \dot{\mathrm{M}} \\
{\left[\mathrm{M}_{\odot} \mathrm{yr}^{-1}\right]}\end{array}$} & \multirow[t]{2}{*}{$\begin{array}{c}\log \mathrm{M}_{\text {disk }} \\
{\left[\mathrm{M}_{\odot}\right]}\end{array}$} \\
\hline & $8498 \AA ̊$ & $8542 \AA$ & $8662 \AA$ & $8498 \AA$ & $8542 \AA$ & $8662 \AA$ & $8498 \AA$ & $8542 \AA$ & $8662 \AA$ & & & \\
\hline \multicolumn{13}{|c|}{ Classical T Tauri stars } \\
\hline AA Tau & 1.47 & 4.25 & 3.12 & 25 & 33 & 28 & -1 & 1 & 2 & 16 & -8.48 & -2.00 \\
\hline BP Tau & 6.37 & 8.67 & 6.44 & 27 & 39 & 30 & 1 & 2 & 3 & 15 & -7.54 & -1.70 \\
\hline CW Tau & 11.27 & 11.13 & 9.31 & 184 & 219 & 190 & 11 & 20 & 16 & 38 & -7.61 & -2.70 \\
\hline CY Tau & 6.45 & 8.39 & 6.43 & 34 & 65 & 53 & -2 & -1 & 0 & 14 & -8.12 & -2.22 \\
\hline DF Tau & 2.94 & 3.15 & 2.40 & 61 & 66 & 56 & -1 & -1 & 0 & 33 & -7.62 & -3.40 \\
\hline DG Tau & 38.77 & 38.04 & 35.17 & 196 & 213 & 191 & 4 & 4 & 4 & 41 & -6.30 & -1.70 \\
\hline DK Tau & 6.85 & 6.80 & 6.44 & 140 & 166 & 142 & -2 & -10 & 1 & 18 & -7.42 & -2.30 \\
\hline DL Tau & 53.52 & 52.37 & 43.04 & 234 & 268 & 241 & 6 & 6 & 4 & 15 & -6.79 & -1.05 \\
\hline DO Tau & 28.02 & 30.16 & 19.56 & 126 & 137 & 119 & -3 & -1 & 0 & 18 & -6.85 & -2.15 \\
\hline DR Tau & 25.94 & 27.13 & 24.15 & 108 & 158 & 126 & 1 & -6 & 3 & 116 & -6.50 & -1.70 \\
\hline FN Tau & 1.17 & 1.95 & 1.25 & 17 & 26 & 19 & 0 & 1 & 2 & 10 & & \\
\hline FP Tau & 0.66 & 1.11 & 0.97 & 54 & 58 & 51 & -21 & -20 & -18 & 46 & & \\
\hline FX Tau & 0.67 & 1.73 & 1.25 & 23 & 29 & 21 & 0 & 0 & 3 & 13 & -8.65 & -3.05 \\
\hline GI Tau & 1.19 & 2.30 & 1.68 & 22 & 27 & 22 & -1 & 0 & 2 & 16 & -8.02 & \\
\hline GK Tau & 1.70 & 2.16 & 1.85 & 39 & 44 & 36 & 2 & 3 & 5 & 27 & -8.19 & -2.70 \\
\hline HL Tau & 31.70 & 31.80 & 25.20 & 148 & 175 & 163 & & & & & & -1.22 \\
\hline HN Tau & 50.96 & 46.66 & 39.21 & 321 & 309 & 276 & -100 & 58 & 64 & & -8.89 & -3.10 \\
\hline LkCa 8 & 4.76 & 5.41 & 3.14 & 28 & 34 & 27 & 2 & 4 & 5 & 16 & -9.10 & -2.52 \\
\hline RW Aur & 69.10 & 68.00 & 53.20 & 245 & 310 & 291 & & & & & -7.12 & -2.40 \\
\hline RY Tau & 3.76 & 2.64 & 2.57 & 250 & 325 & 320 & & & & & -7.11 & -1.70 \\
\hline T Tau & 4.19 & 4.34 & 3.87 & 80 & 83 & 82 & & & & & -7.12 & -2.10 \\
\hline XZ Tau & 8.43 & 8.09 & 6.53 & 91 & 94 & 99 & & & & & & \\
\hline \multicolumn{13}{|c|}{ Transitional disk objects } \\
\hline CoKu Tau 4 & 0.46 & 0.65 & 0.48 & 44 & 50 & 38 & 11 & 10 & 15 & 37 & $<-10.00$ & -3.30 \\
\hline CX Tau & 1.29 & 1.89 & 1.20 & 34 & 40 & 32 & 4 & 5 & 6 & 29 & -8.97 & -3.00 \\
\hline DM Tau & 1.23 & 1.58 & 1.12 & 18 & 25 & 19 & -1 & 0 & 1 & 11 & -7.95 & -1.70 \\
\hline FO Tau & 2.03 & 2.94 & 2.35 & 25 & 37 & 28 & 0 & 1 & 2 & 14 & -7.90 & -3.22 \\
\hline GM Aur & 1.50 & 3.65 & 2.91 & 30 & 43 & 41 & 0 & 1 & 2 & 20 & -8.02 & -1.52 \\
\hline LkCa 15 & 0.91 & 1.23 & 0.96 & 25 & 30 & 11 & 2 & 4 & 6 & 18 & -8.87 & -1.30 \\
\hline UX Tau & 0.63 & 1.06 & 0.89 & 41 & 46 & 37 & -2 & -1 & 1 & 35 & -9.00 & -2.30 \\
\hline V773 Tau & $<0.94$ & 1.68 & 1.36 & 67 & 92 & 71 & -37 & -19 & -30 & 58 & -9.62 & -3.30 \\
\hline V836 Tau & 1.70 & 5.20 & 3.06 & 31 & 41 & 32 & 0 & 0 & 2 & 18 & -8.98 & -2.00 \\
\hline \multicolumn{13}{|c|}{ Weak-line T Tauri stars } \\
\hline HBC 374 & 1.54 & 2.11 & 0.70 & 32 & 37 & 31 & -1 & 1 & 1 & 24 & $<-7.78$ & $<-3.40$ \\
\hline HD 283716 & & & & & & & 74 & 73 & 72 & & & \\
\hline NTTS $032461+2420$ & 0.46 & 1.20 & 0.64 & 89 & 140 & 22 & -32 & -31 & -53 & & & \\
\hline NTTS $041559+1716$ & 1.83 & 4.36 & 1.58 & 133 & 135 & 101 & 9 & 4 & -17 & 107 & $<-8.92$ & $<-3.49$ \\
\hline NTTS $042417+1744$ & 0.29 & 0.43 & 0.36 & 24 & 26 & 21 & 8 & 4 & 7 & 23 & $<-8.03$ & $<-3.52$ \\
\hline RX J0405.3+2009 & 0.37 & 0.51 & 0.36 & 34 & 34 & 31 & 5 & 2 & 5 & 39 & & \\
\hline RX J0409.2+1716 & 1.02 & 1.68 & 1.00 & 99 & 104 & 100 & 19 & 36 & 10 & & & \\
\hline RX J0438.6+1546 & 0.57 & 0.88 & 0.71 & 38 & 41 & 34 & 6 & 4 & 2 & 38 & & \\
\hline RX J0452.5+1730 & 0.59 & 0.73 & 0.49 & 20 & 23 & 19 & 3 & 1 & 1 & 14 & & \\
\hline RX J0459.7+1430 & 0.44 & 0.75 & 0.43 & 23 & 26 & 22 & 3 & 5 & 3 & 22 & & \\
\hline V410 Tau & 0.21 & 0.43 & 0.27 & 51 & 54 & 52 & & & & & $<-8.42$ & $<-3.40$ \\
\hline V819 Tau & 1.44 & 2.73 & 2.44 & 25 & 36 & 28 & 0 & 2 & 3 & 14 & $<-8.48$ & $<-3.40$ \\
\hline V827 Tau & 1.76 & 2.12 & 1.83 & 37 & 41 & 34 & 1 & 2 & 4 & 28 & $<-8.15$ & $<-3.52$ \\
\hline V830 Tau & 0.56 & 0.88 & 0.58 & 52 & 52 & 47 & -21 & -15 & -17 & 43 & $<-8.10$ & $<-3.52$ \\
\hline
\end{tabular}

objects. As a reference, we also show the equivalent widths of the lines of ZAMSs reported by Marsden et al. (2009), in which 24 solar-type stars in the IC 2391 cluster and 28 stars in the IC 2602 cluster are investigated. Among the sample, CTTSs show the strongest emissions, although there is a large scatter in the equivalent widths. The emission lines of the WTTSs and ZAMSs are weak. A similar trend was reported in Hamann \& Persson (1992b), in which T Tauri stars with large infrared excess show strong $\mathrm{Ca}$ II IRT emission. Figure 3 shows the correlation between the FWHMs of the Ca II IRT lines and their equivalent widths. Lines with stronger emissions are broader. Batalha et al. (1996) proposed that the narrow line emits from the hot chromosphere, while Hartmann et al. (1994) claimed that the broad line is formed at magnetosphere in which circumstellar material falls onto the central star. A similar but weaker correlation was also found in the $\mathrm{H} \alpha$ emission line (Reipurth et al. 1996).

It is widely accepted that a strong dynamo process is caused by a rapidly rotating photosphere. Figure 4 shows the relationship between the rotational velocities $(v \sin i)$ and the equivalent widths of 


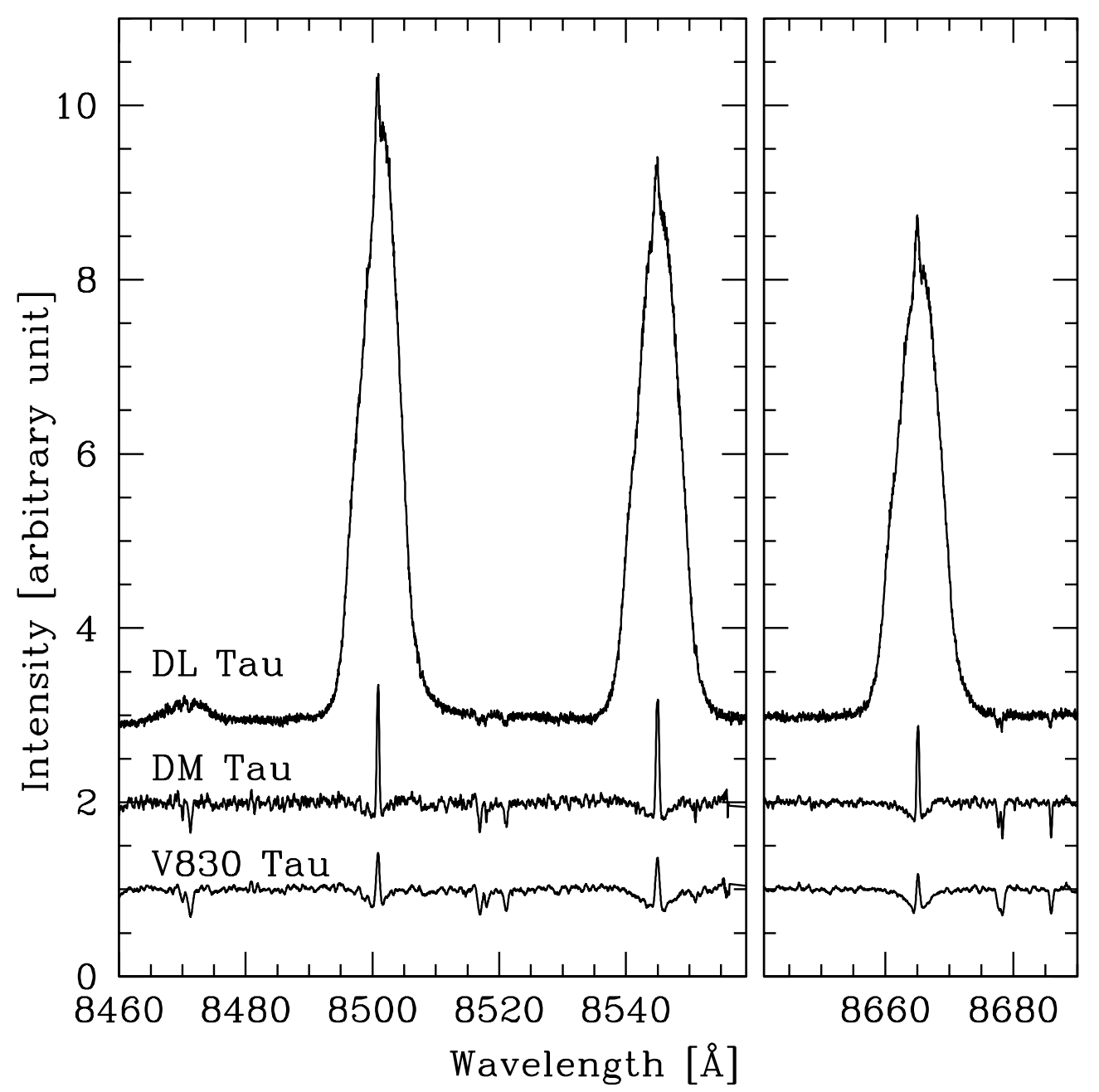

Fig. 1 Ca II IRT emissions of YSOs. DL Tau, a CTTS, has prominent emission features. DM Tau, a transitional disk object, and V830 Tau, a WTTS, show narrow and weak emission lines superimposed on broad absorption features. Photospheric absorption lines are not subtracted.

the Ca II IRT emission lines. We see no correlation in these parameters. Bouvier (1990) and Neuhaeuser et al. (1995) pointed out that WTTSs rotate faster than CTTSs. Therefore, if chromospheric activity is induced by the dynamo process, and if the Ca II IRT emission lines are of chromospheric origin, then the Ca II IRT emission lines of WTTSs are expected to be stronger than those of CTTSs. Because the Ca II IRT emission lines of the WTTSs are not stronger than those of the CTTSs, it does not appear that the strong Ca II emission lines observed in CTTSs are mainly generated in active chromosphere induced by the dynamo process.

It is also well known that CTTSs usually have high rates of mass accretion from their circumstellar disks. Figure 5 shows the relationship between mass accretion rate and the equivalent widths of the $\mathrm{Ca}$ II IRT emissions. We used the mass accretion rates from Gullbring et al. (1998), Hartmann et al. (1998), and White \& Ghez (2001). Gullbring et al. (1998) conducted intermediate-resolution spectrophotometry 


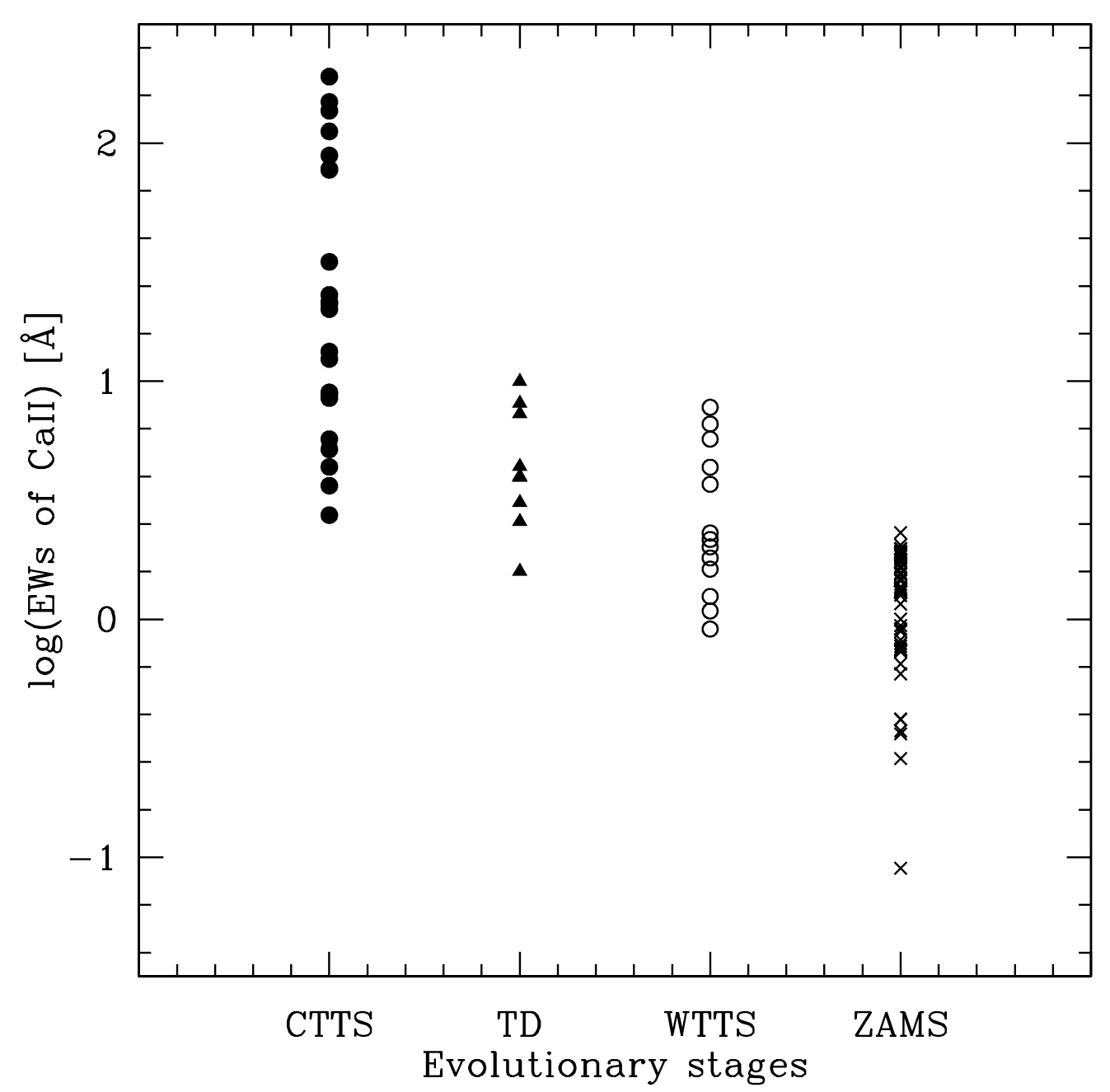

Fig. 2 Equivalent widths of the Ca II IRT emission lines as a function of the evolutional sequence. The vertical axis represents the sum of the equivalent widths of the 3 emission lines. As seen in Figure 1, CTTSs show large equivalent widths.

from 3200 to $5200 \AA$ for 26 CTTSs and 3 WTTSs. The veiling effect of a hot continuum caused by mass accretion phenomena is not negligible in the $U$ - and $B$-bands. The amount of the veiling was estimated by measuring the line-to-continuum ratio of a number of absorption features of the YSO spectrum and the ratios of a template dwarf spectrum. The line-to-continuum ratio was the flux ratio of an absorption line and its adjacent continuum. Then, they estimated the temperature of the hot continuum region by comparing the fluxes at $3600 \AA, 4000 \AA$, and $4750 \AA$. They assumed a constant temperature. The extinction of the object was estimated from broad-band photometry, then they derived the luminosity of the hot continuum region, i.e., an accretion luminosity. Finally, assuming a disk inner radius of 5 stellar radius, they estimated the mass accretion rates. They also proposed the relationship between the $U$-band excess and the accretion luminosity determined from optical veiling estimate. Hartmann et al. (1998) and White \& Ghez (2001) calculated the mass accretion rates using this relationship. From figure 


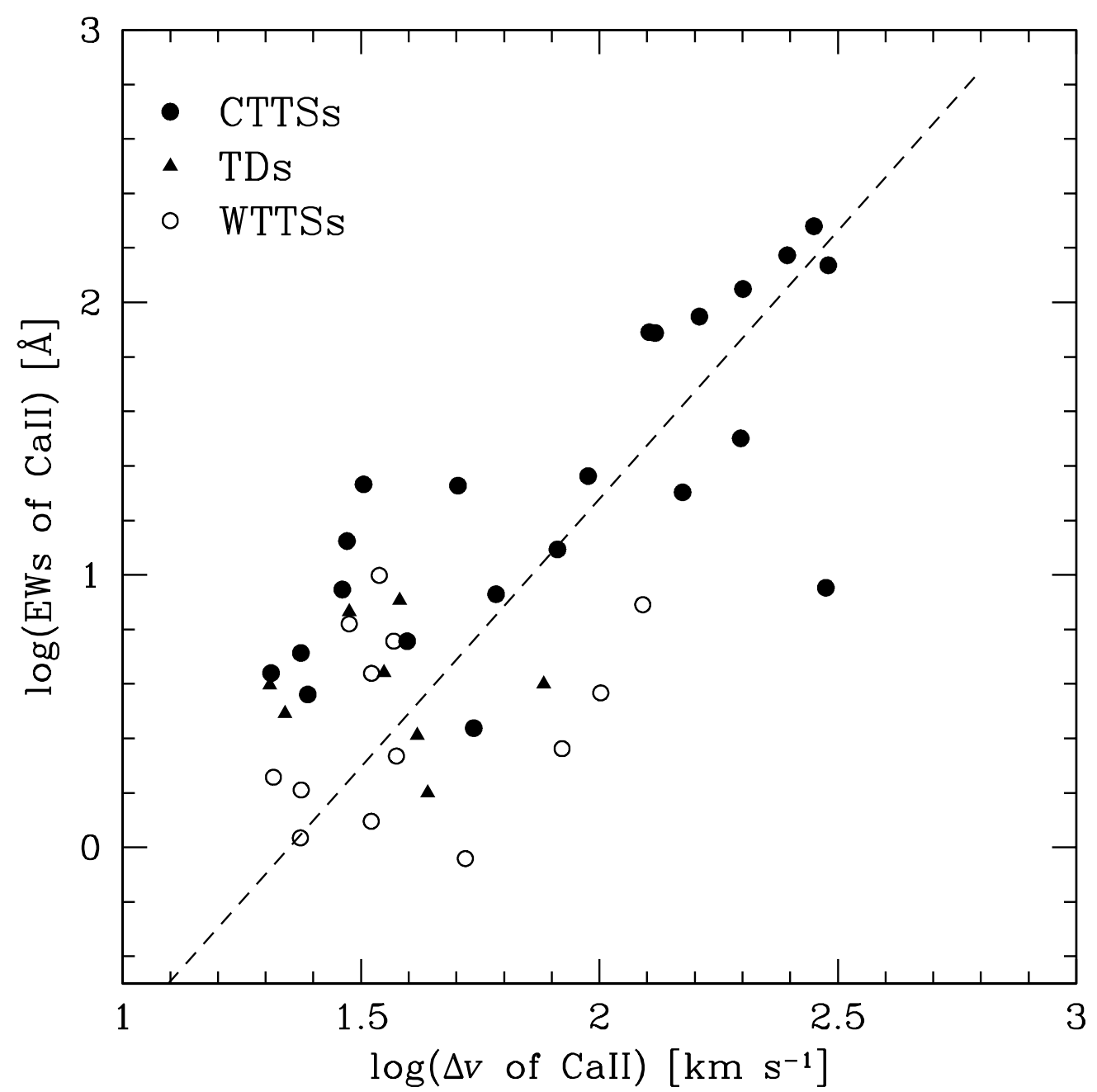

Fig. 3 The equivalent widths of the Ca II IRT emissions as a function of the line widths of the emission lines. The vertical axis represents the sum of the equivalent widths of the 3 emission lines. The horizontal axis means the average of the FWHMs of the 3 emission lines. The dashed line indicates that the equivalent widths increase as the square of the line widths.

5 we noticed a correlation between the mass accretion rates and the equivalent widths of the Ca II IRT emission lines; objects with high mass accretion rates have strong emissions of the Ca II IRT. Models of magnetospheric accretion are proposed to explain broad components of the emission lines observed in CTTSs. In the model, the magnetic field connecting between the photosphere and the circumstellar disk is expected (e.g. Uchida \& Shibata 1985).

The material is accreted from the circumstellar disk along the magnetic field lines. We investigated the correlation between the strengths of the Ca II IRT emission lines and the mass of the circumstellar disk (Figure 6). We used the disk mass from Andrews \& Williams (2005). They conducted an extensive submillimeter continuum survey of YSOs in the Taurus-Auriga star forming region. For the objects whose mid- and far-infrared photometric data were available, they fitted the spectrum of the model 


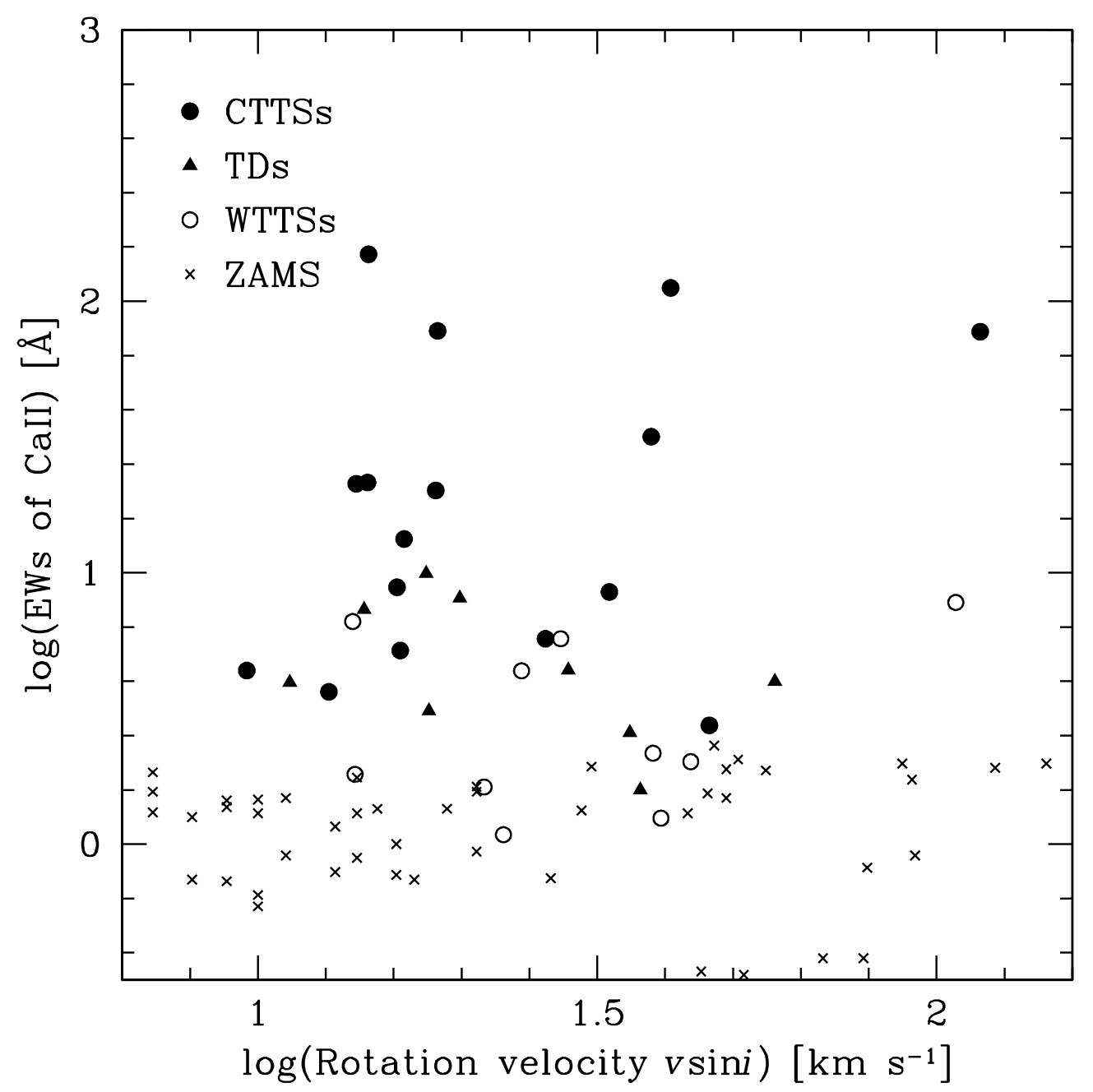

Fig. 4 The equivalent widths of the Ca II IRT emissions of YSOs as a function of photospheric rotation velocity. The vertical axis represents the sum of the equivalent widths of the 3 emission lines. There is no apparent correlation in these parameters.

disk between mid-infrared and submillimeter wavelengths, then determined the disk mass. For the other objects, the disk mass was estimated from the submillimeter flux. We found that the equivalent widths of the Ca II IRT emission lines of the transitional disk objects are one-tenth the value of those of CTTSs, even if the masses of the circumstellar disks are comparable. It is considered that the transitional disk objects have an inner hole with a radius of a few AU or a few tens of AU. An object exhibits infrared excess if its disk fills such an inner region. Figure 7 shows the equivalent widths of the Ca II IRT emission lines as a function of near-infrared $J-K$ color. Photospheric color of the object and reddening caused by interstellar material are subtracted, so that the color in the figure indicates near-infrared excess caused by an inner region of the circumstellar disk. The CTTSs show large variety in the near-infrared excess, whereas the transitional disk objects and the WTTSs have little near-infrared excesses. The objects with large near-infrared excess show strong Ca II IRT emissions. This correlation supports an 


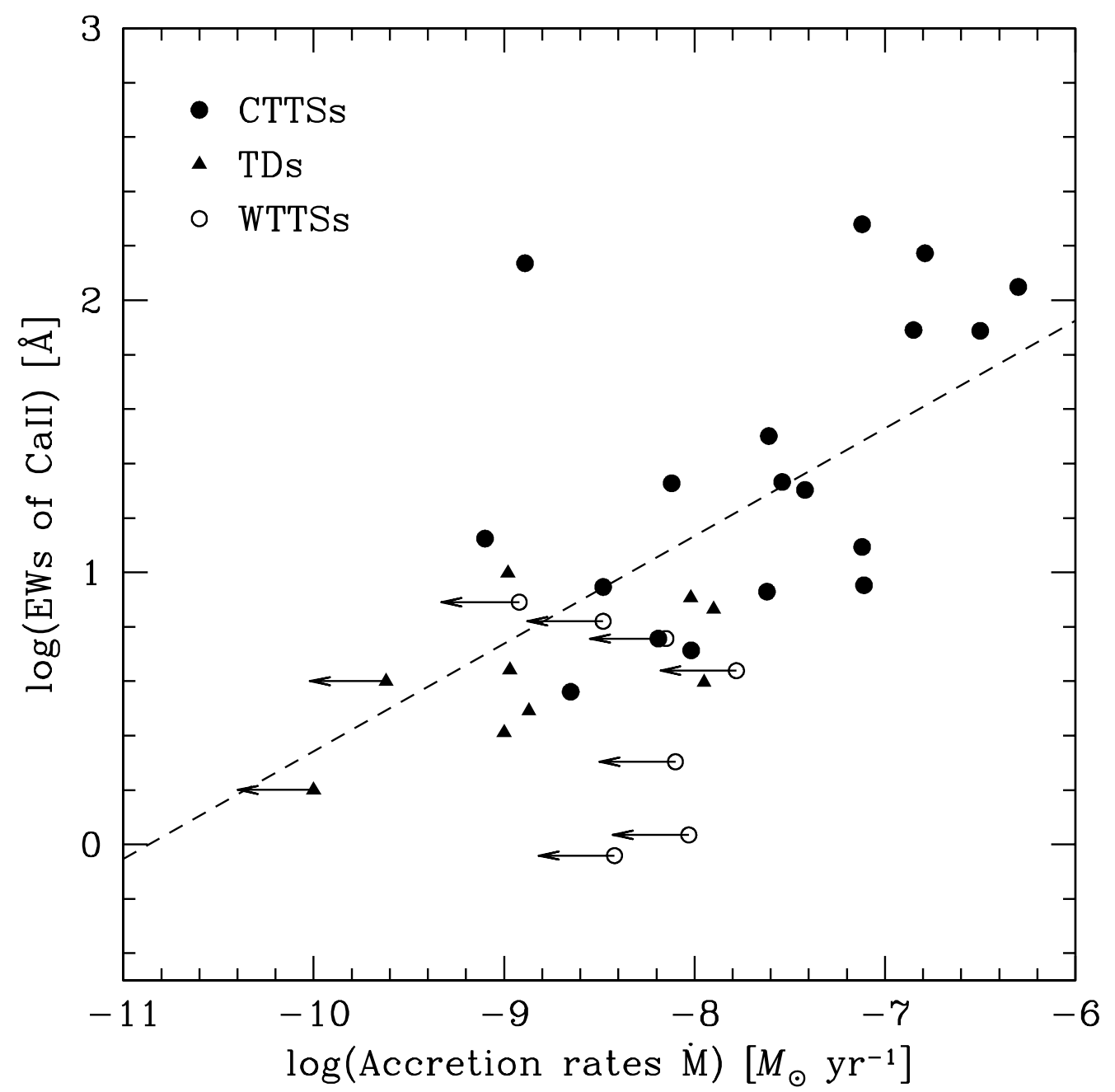

Fig. 5 The equivalent widths of the Ca II IRT emission lines of YSOs as a function of mass accretion rate of the circumstellar materials. The vertical axis represents the sum of the equivalent widths of the 3 emission lines. The dashed line means that the equivalent widths increase as the mass accretion rates to the power of 0.4.

idea that materials accreting from the warmer inner disk enhance the stellar activity. We also suggest that the magnetic fields between the photosphere and the circumstellar disk are already disconnected at the evolution phase of the transitional disk objects.

The line widths of the Ca II IRT emissions also differ significantly between the CTTSs and the transitional disk objects. Figure 8 shows the line widths of the Ca II IRT emissions as a function of the rotational velocity of the photosphere. The rotational velocity is estimated from the line width of the absorption line. As shown in the figure, the objects can be classified into two groups. One group consists of the objects for which the widths of the Ca II IRT emission lines are comparable to, or slightly larger than, the width of the absorption line. The most CTTSs as well as all transitional disk objects and WTTSs are classified into this group. We consider that the emission lines emanate from the stellar 


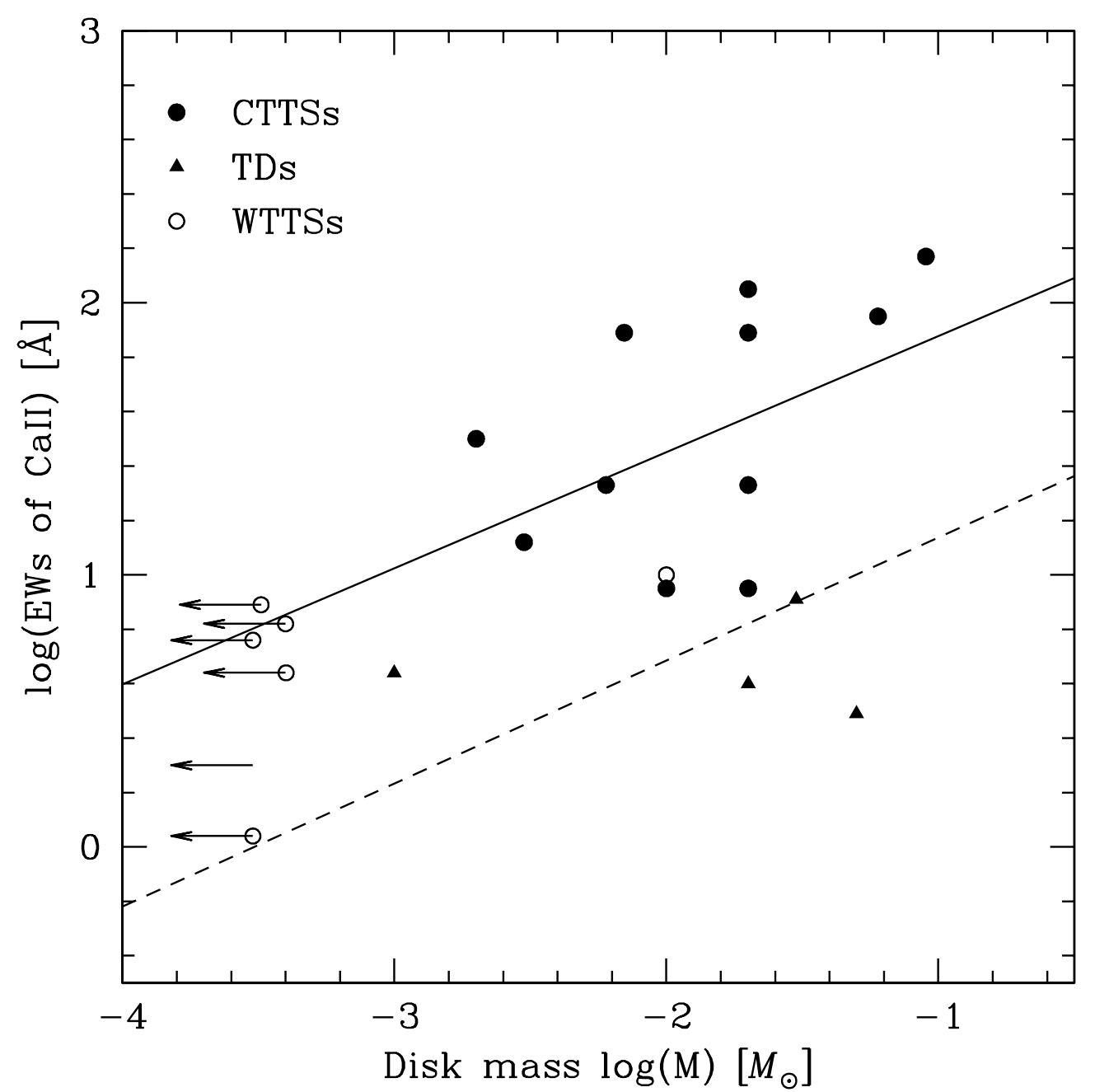

Fig. 6 The equivalent widths of the Ca II IRT emission lines of YSOs as a function of mass of the circumstellar disks. The vertical axis represents the sum of the equivalent widths of the 3 emission lines. Least-squares fits are plotted for the CTTS sample (solid line) and the transitional disk object sample (dashed line). Both samples consist of single stars.

chromosphere for the objects in this group. The other group consists of the exceptions. All objects in this group are CTTSs (CW Tau, DG Tau, DK Tau, DL Tau, and DO Tau). The widths of the emission lines are significantly broad, compared to the line widths of the photospheric absorption line. We found that the objects in this group have large mass accretion rates $\left(\dot{\mathrm{M}}>10^{-8} \mathrm{M}_{\odot} \mathrm{yr}^{-1}\right.$, see table 2$)$. We consider that the Ca II IRT emission lines of the objects in this group emanate from the magnetosphere between the photosphere and the circumstellar disk. Muzerolle et al. (1998) observed 11 CTTSs with a spectral resolution $R \sim 35,000$. Several emission lines including the Ca II IRT emission lines were detected. They classified the objects into three groups based on the shapes of the Ca II IRT emission lines. One is the objects with a narrow emission line superimposed on the photospheric broad absorption. The second group consists of the objects showing both broad and narrow component emissions. The objects 


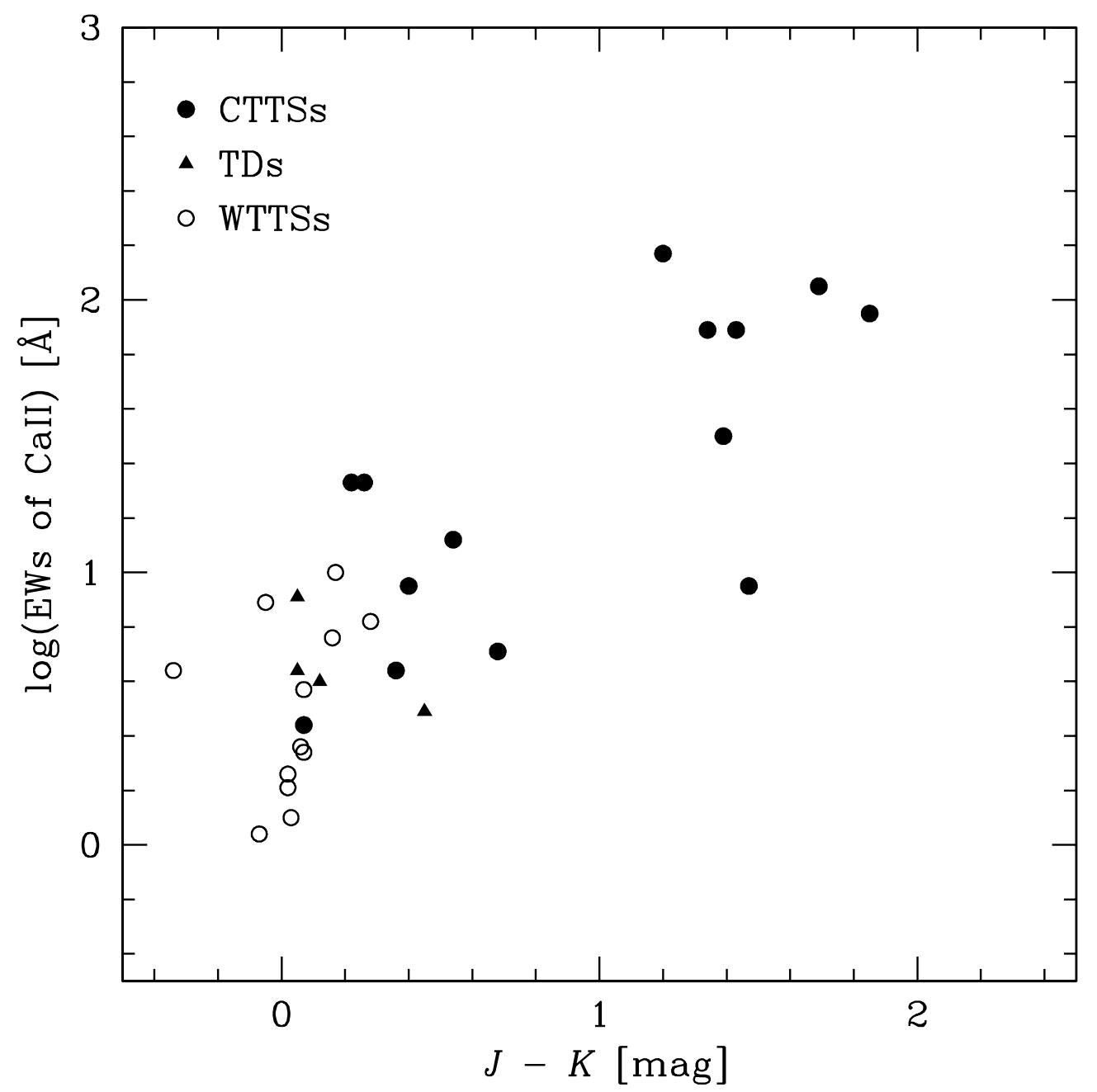

Fig. 7 The equivalent widths of the Ca II IRT emission lines of YSOs as a function of nearinfrared excess. The vertical axis represents the sum of the equivalent widths of the 3 emission lines. The sample consists of single stars.

belong to the third group show only a broad-component emission. Hamann \& Persson (1992a) proposed that the broad components of the Ca II IRT emission emanates from an extended envelope with large turbulent velocities. Muzerolle et al. (1998) constructed the magnetospheric model. The magnetospheric emission line is characterized by a large line width, a blueshifted asymmetry, and a slightly blueshifted peak. They claimed that the Ca II IRT emission line profiles of a part of the objects in the third group are well reproduced by the magnetospheric model. Among the exceptions in our sample (CW Tau, DG Tau, DK Tau, DL Tau, and DO Tau), DG Tau and DO Tau exhibit the emission line profiles similar to that given by the magnetospheric model. However, the line profiles of the rest objects are complicated and seem not to be reproduced by the magnetospheric model. Moreover, by comparing our spectra to the spectra presented in Muzerolle et al. (1998), we find significant difference in the line profiles between the spectra. This difference may indicate variability of the emission lines. Because the spatial sizes of 


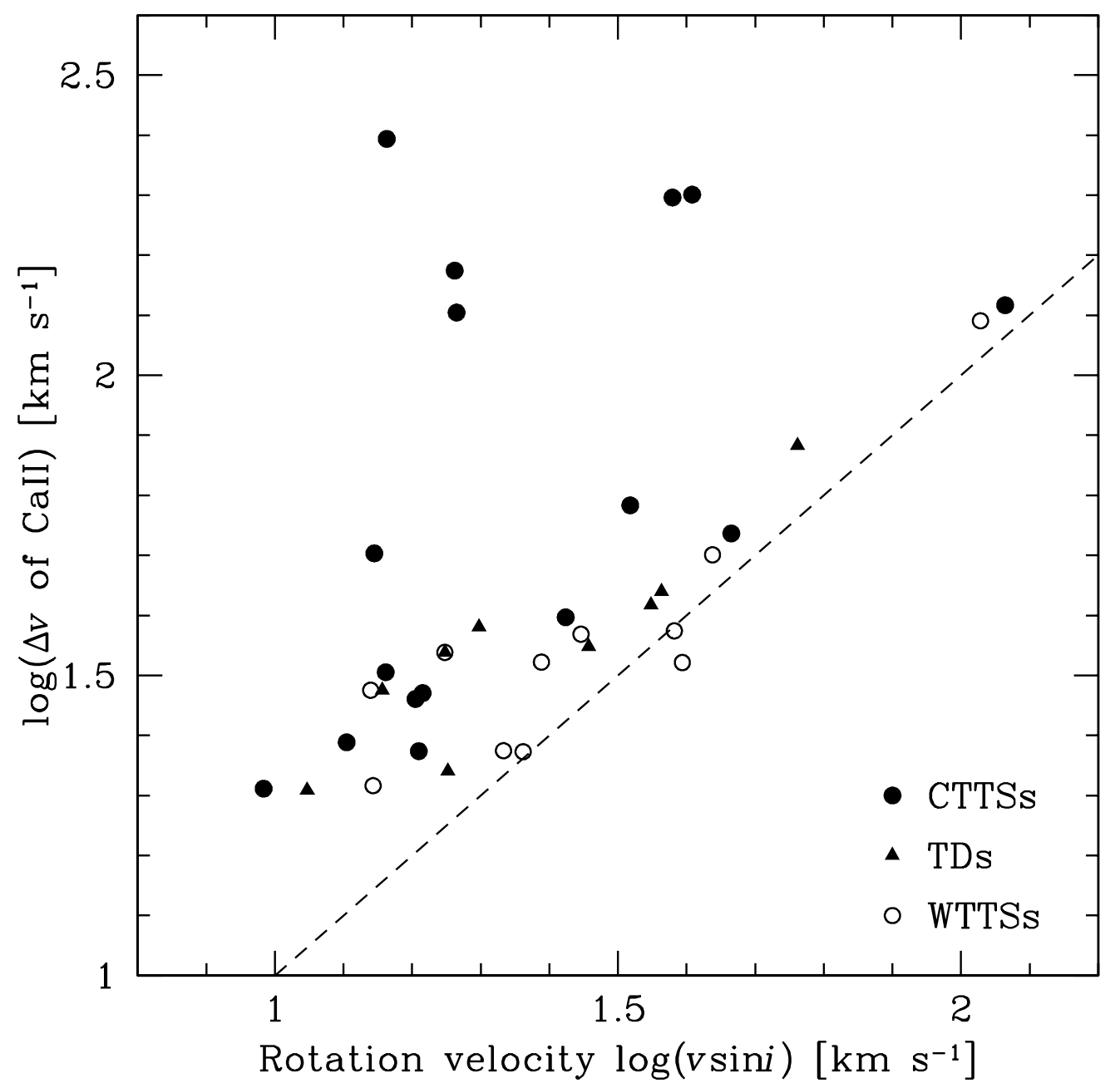

Fig. 8 FWHM of the Ca II IRT emissions as a function of the FWHMs of the photospheric absorption line. The dashed line represents the case where the FWHMs of both lines are same.

the chromosphere and the magnetosphere are different, time-series spectroscopy with short intervals will identify whether the origin of the broad and strong emission lines of the Ca II IRT is the turbulent chromosphere or the magnetosphere.

\section{CONCLUSIONS}

We measured strengths and line profiles of Ca II infrared triplet emission lines with high-resolution optical spectra of 39 young stellar objects.

1. The equivalent widths of the emission lines decrease with stellar evolution.

2. The CTTSs with high mass accretion rates show strong and broad emission of the Ca II lines. It is considered that the lines emit from the turbulent chromosphere or the magnetosphere between the photosphere and the circumstellar disk. 
3. The transitional disk objects and the WTTSs show weak and narrow emission of the Ca II lines. The line widths are comparable to, or slightly larger than, the line width of the photospheric absorption line. The emission lines are attributed to the chromospheric activity. The equivalent widths of $\mathrm{Ca}$ II lines of the transitional disk objects are one-tenth of those of classical T Tauri stars, even if the masses of the circumstellar disks are comparable. Mass accretion from the warmer inner disk induces chromospheric and/or magnetospheric activities.

Acknowledgements We thank the telescope staff members and operators at the Subaru Telescope. This research has made use of the Keck Observatory Archive (KOA), which is operated by the W. M. Keck Observatory and the NASA Exoplanet Science Institute (NExScI), under contract with the National Aeronautics and Space Administration. 
Appendix A: SPECTRA OF THE YSOS

High resolution spectra of 39 YSOs are presented in the appendix.

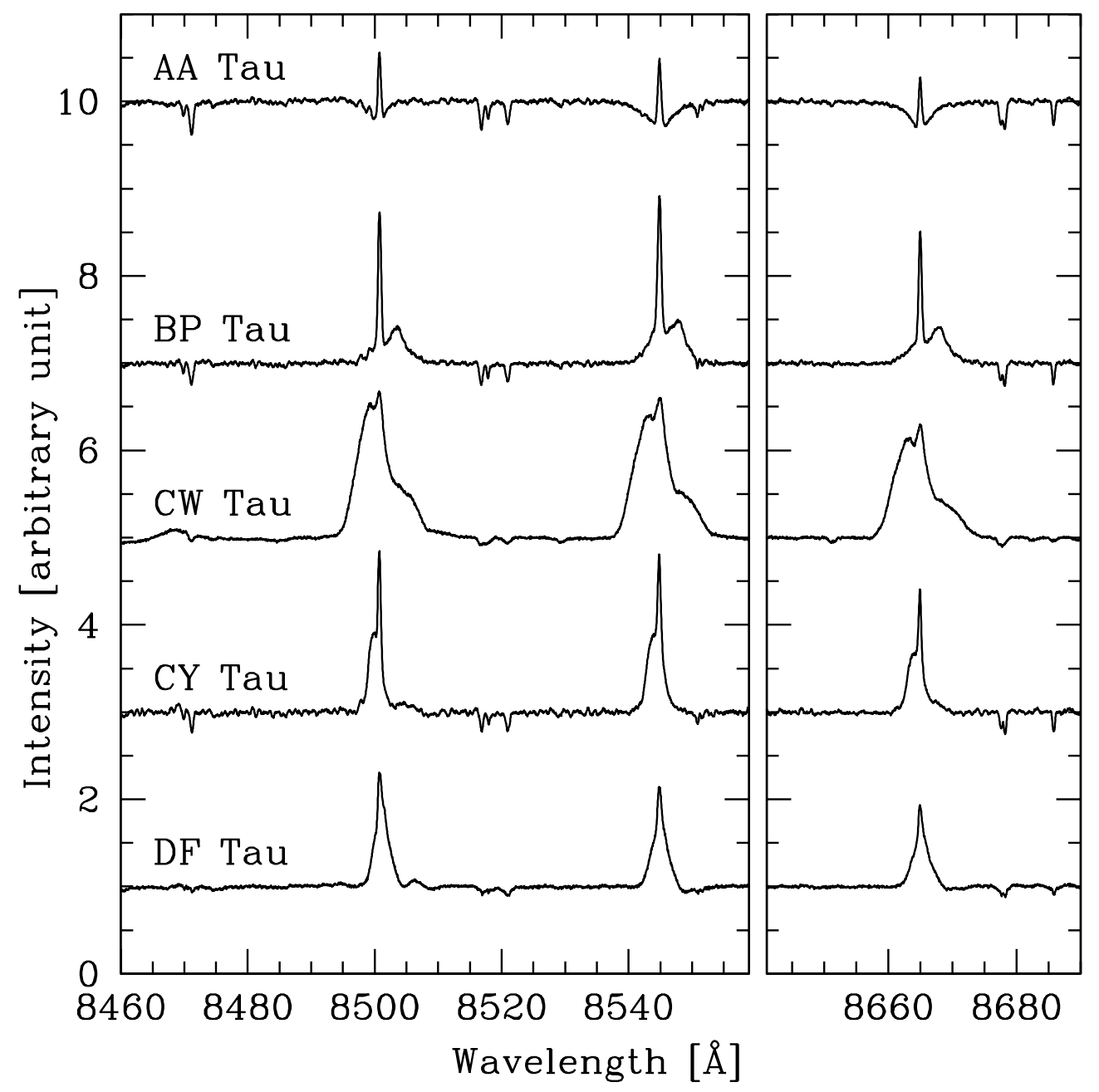

Fig. A.1 High resolution spectra of the YSOs. 


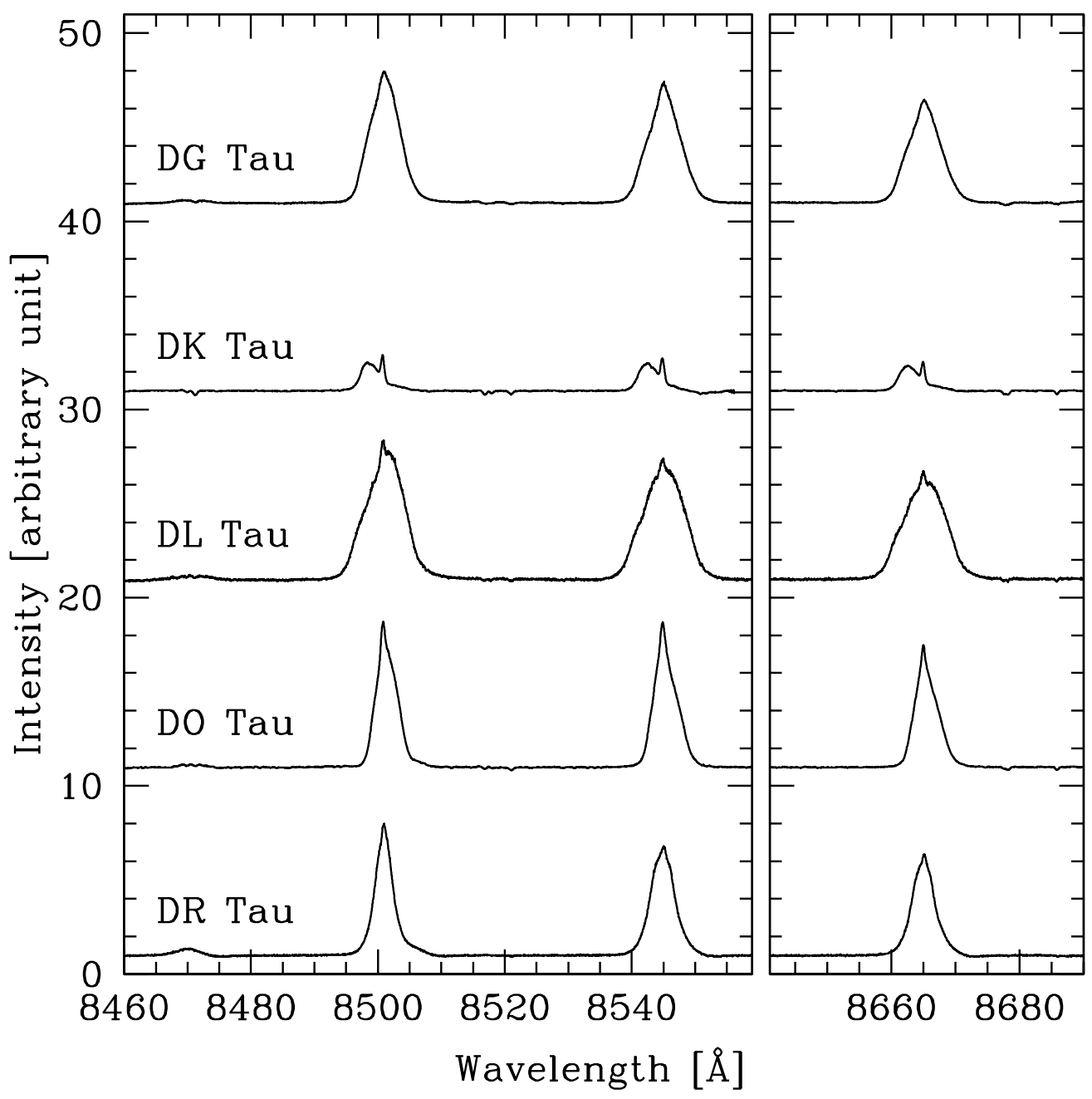

Fig. A.2 High resolution spectra of the YSOs. 


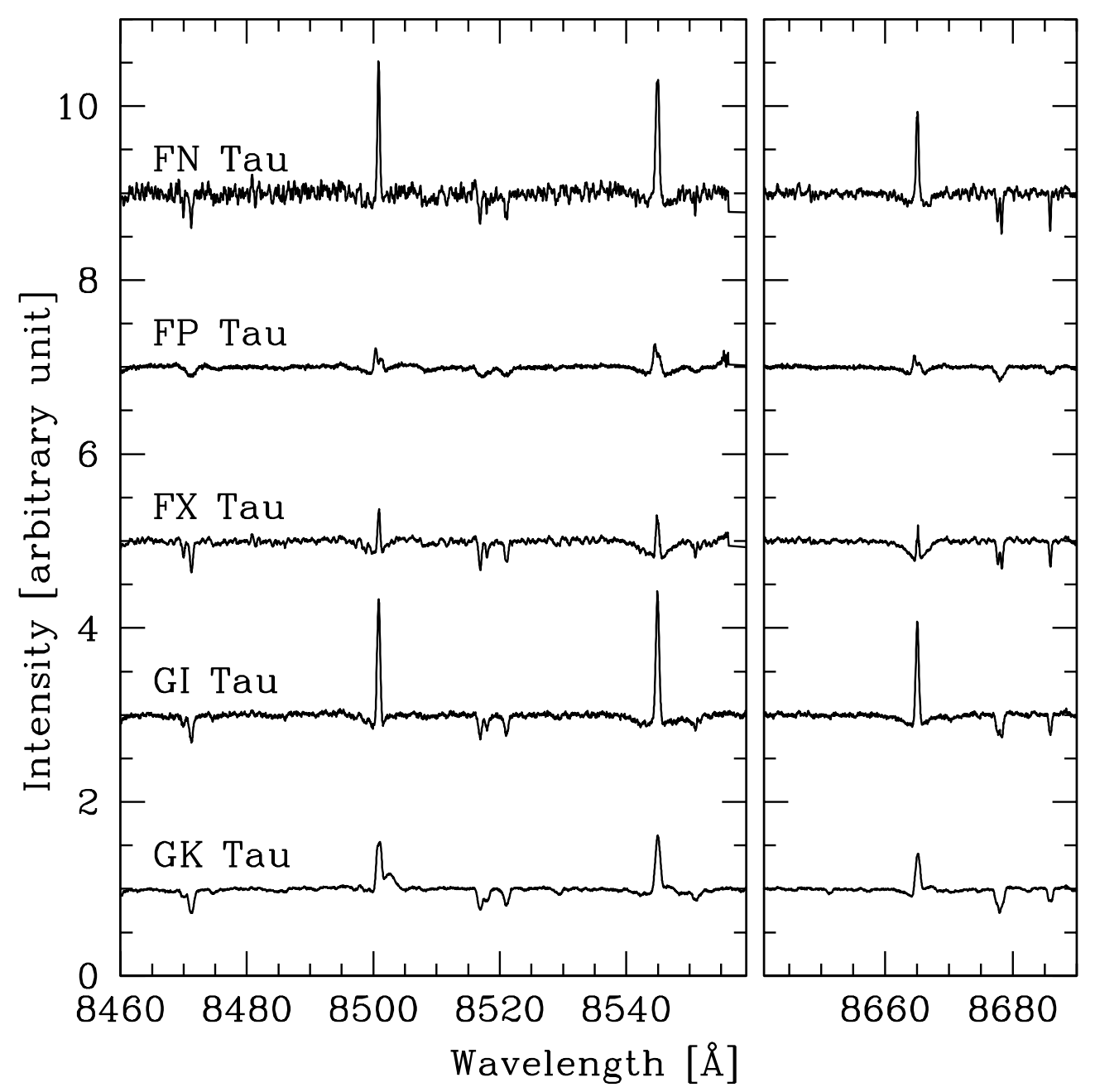

Fig. A.3 High resolution spectra of the YSOs. 


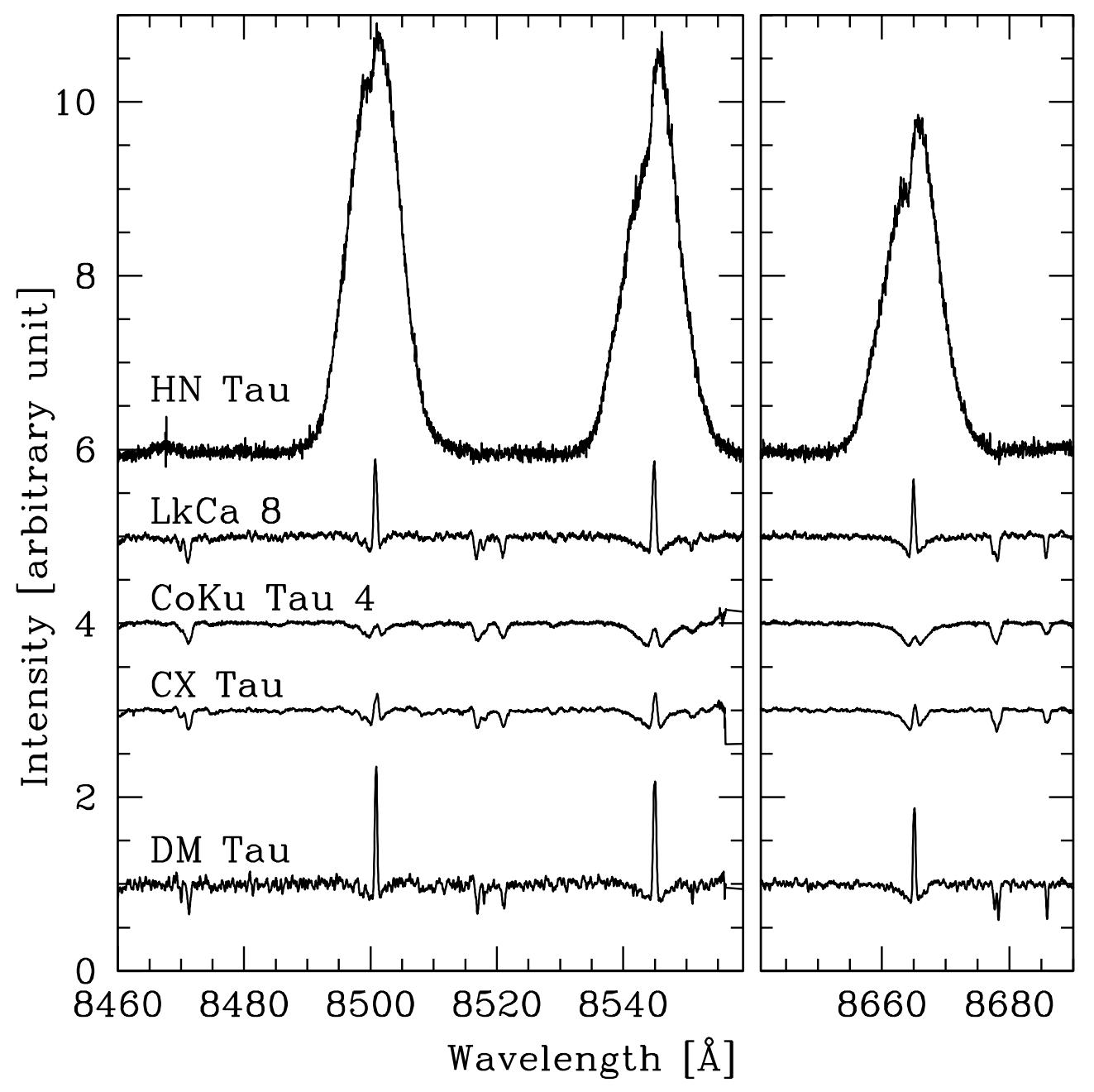

Fig. A.4 High resolution spectra of the YSOs. 


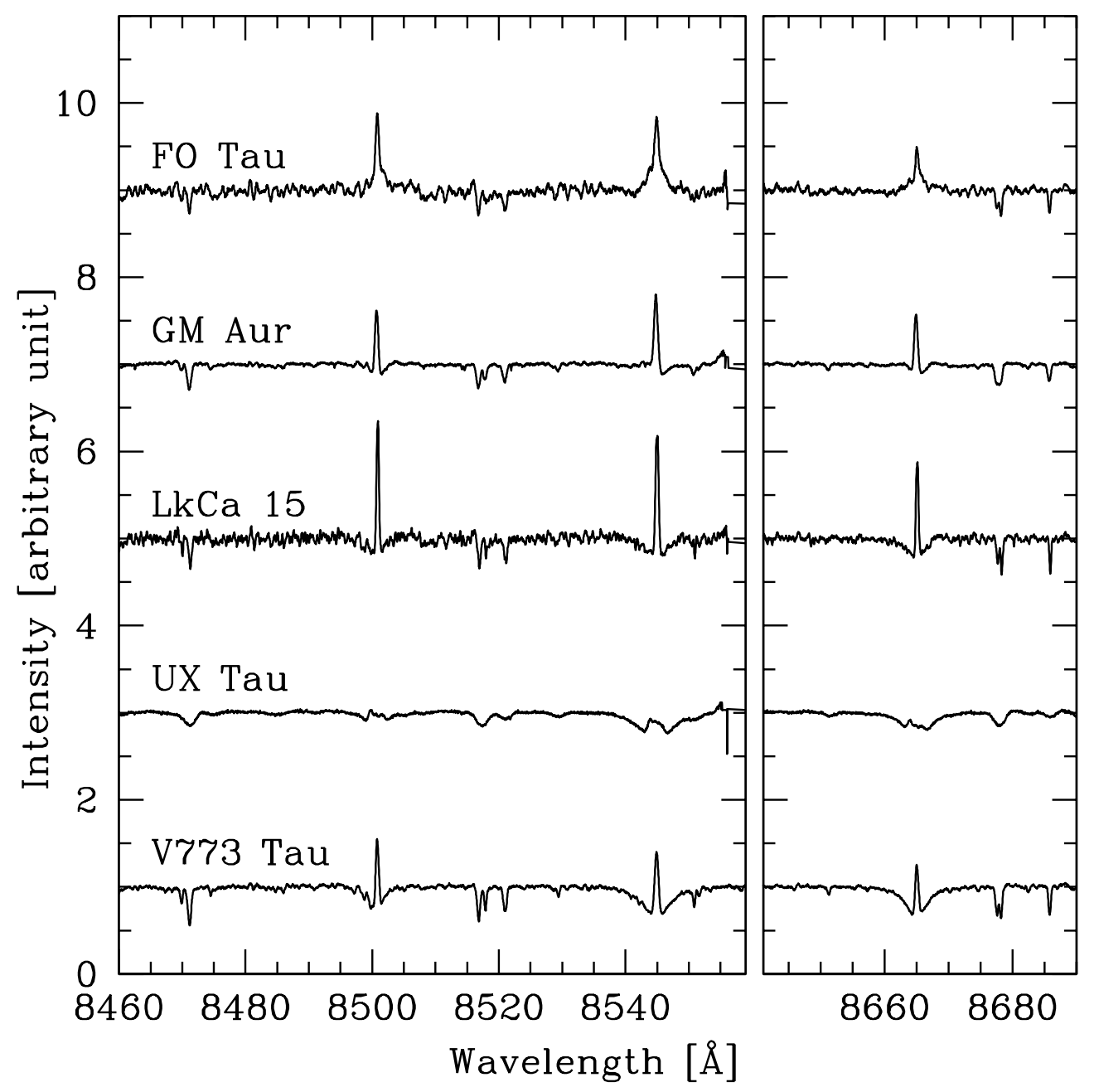

Fig. A.5 High resolution spectra of the YSOs. 


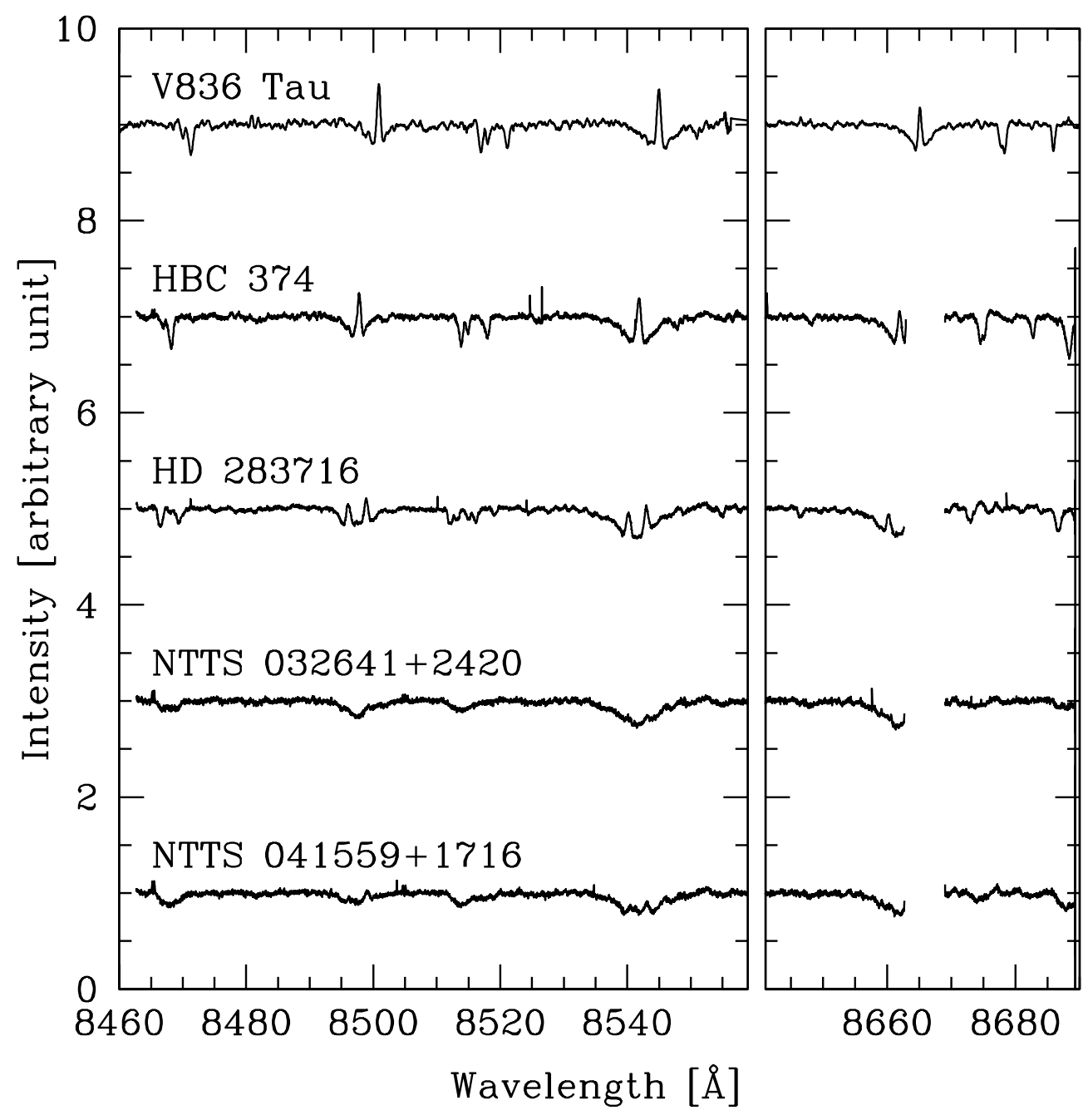

Fig. A.6 High resolution spectra of the YSOs. 


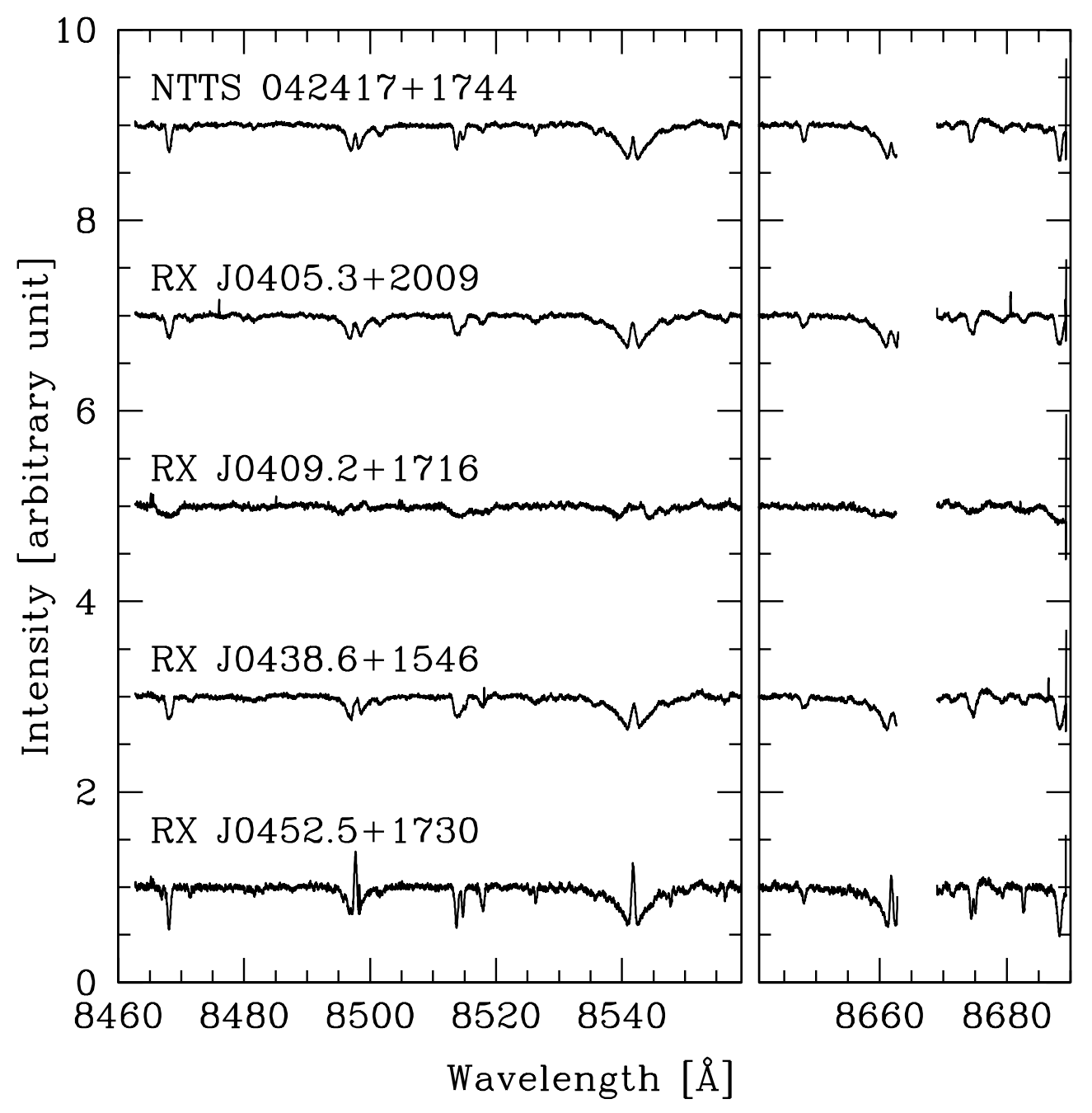

Fig. A.7 High resolution spectra of the YSOs. 


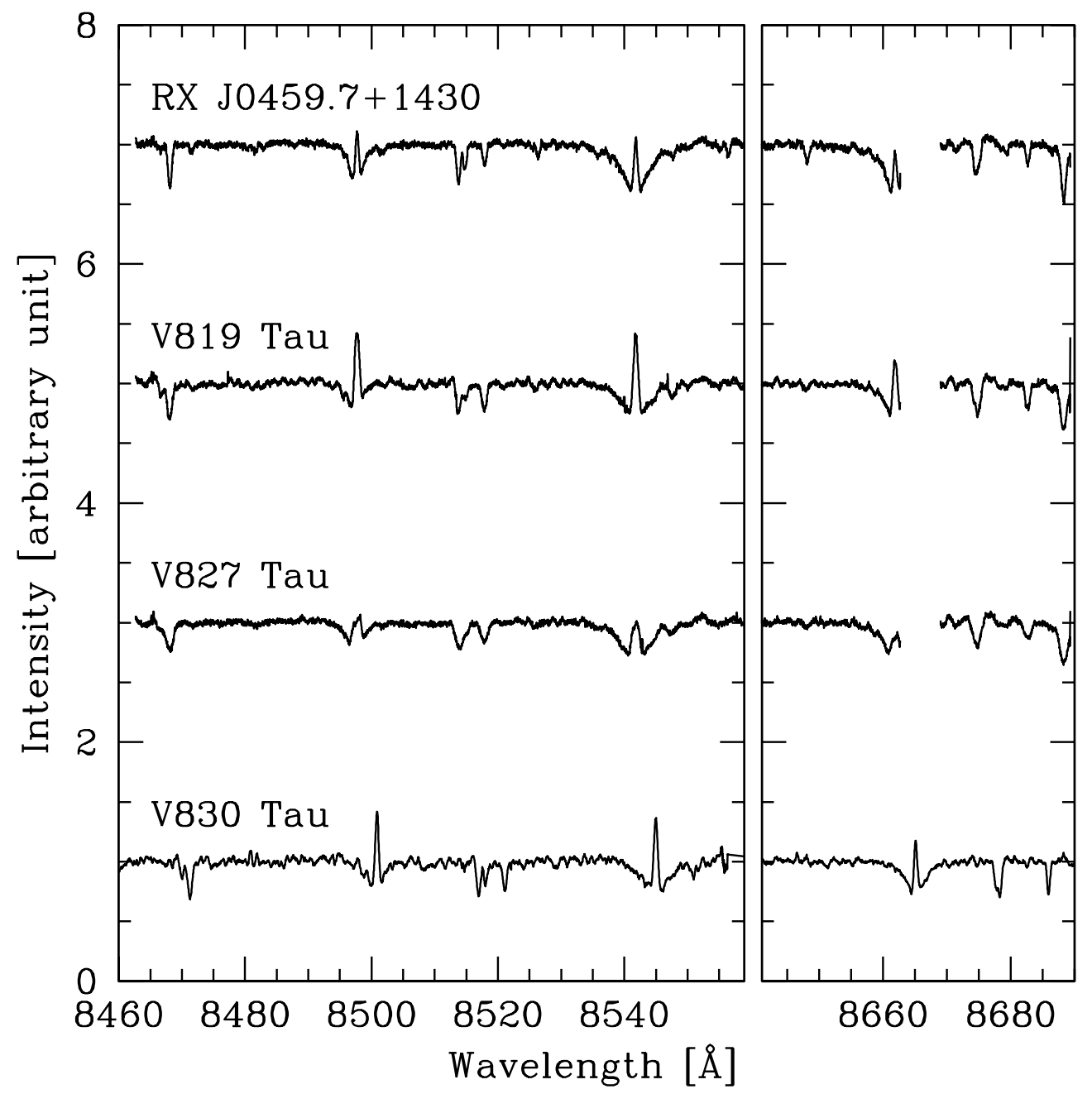

Fig. A.8 High resolution spectra of the YSOs. 


\section{References}

Andrews, S. M., \& Williams, J. P. 2005, ApJ, 631, 1134

Batalha, C. C., Stout-Batalha, N. M., Basri, G., \& Terra, M. A. O. 1996, ApJS, 103, 211

Bertout, C., Basri, G., \& Bouvier, J. 1988, ApJ, 330, 350

Bouvier, J. 1990, AJ, 99, 946

Ghez, A. M., White, R. J., \& Simon, M. 1997, ApJ, 490, 353

Gullbring, E., Hartmann, L., Briceno, C., \& Calvet, N. 1998, ApJ, 492, 323

Hamann, F., \& Persson, S. E. 1992a, ApJS, 82, 247

Hamann, F., \& Persson, S. E. 1992b, ApJ, 394, 628

Hartmann, L., Hewett, R. \& Calvet, N. 1994, ApJ,

Hartmann, L., Calvet, N., Gullbring, E., \& D’Alessio, P. 1998, ApJ, 495, 385

Ireland, M. J., \& Kraus, A. L. 2008, ApJ, 678, L59

Katsukawa, Y., Berger, T. E., Ichimoto, K., et al. 2007, Science, 318, 1594

Kohler, R., \& Leinert, C. 1998, A\&A, 331, 977

Leinert, C., Zinnecker, H., Weitzel, N., et al. 1993, A\&A, 278, 129

Marsden, S. C., Carter, B. D., \& Donati, J.-F. 2009, MNRAS, 399, 888

Muzerolle, J., Hartmann, L., \& Calvet, N. 1998, AJ, 116, 455

Neuhaeuser, R., Sterzik, M. F., Schmitt, J. H. M. M., Wichmann, R., \& Krautter, J. 1995, A\&A, 297, 391

Noyes, R. W., Hartmann, L. W., Baliunas, S. L., Duncan, D. K., \& Vaughan, A. H. 1984, ApJ, 279, 763

Reipurth, B., Pedrosa, A., \& Lago, M. T. V. T. 1996, A\&AS, 120, 229

Sartoretti, P., Brown, R. A., Latham, D. W., \& Torres, G. 1998, A\&A, 334, 592

Takagi, Y., Itoh, Y., Oasa, Y., \& Sugitani, K. 2011, PASJ, 63, 677

Uchida, Y., \& Shibata, K. 1985, PASJ, 37, 515

White, R. J., \& Ghez, A. M. 2001, ApJ, 556, 265 